\title{
Les corps en perspectives
}

Nathalie Frogneux

\section{(2) OpenEdition}

Journals

Édition électronique

URL : http://journals.openedition.org/pa/302

DOI : $10.4000 /$ pa.302

ISSN : 2273-0362

\section{Éditeur}

Université Lumière Lyon 2

\section{Édition imprimée}

Pagination : 8-23

ISBN : 1634-7706

ISSN : 1634-7706

Référence électronique

Nathalie Frogneux, «Les corps en perspectives », Parcours anthropologiques [En ligne], 9 | 2014, mis en ligne le 30 septembre 2014, consulté le 30 avril 2019. URL : http://journals.openedition.org/pa/302 ; DOI : $10.4000 /$ pa.302 


\section{Les corps en perspectives}

Nathalie Frogneux

Centre Europè/ Institut Supérieur de Philosophie LAAP/ IACCHOS

Université catholique de Louvain, Belgique

Posons d'abord nos bases épistémologiques, car elles nous permettent d'emblée d'affirmer le caractère incarné de notre propos. Tout d'abord, nous nous situons dans le cadre de l'anthropologie philosophique occidentale et même dans une tradition phénoménologique et de philosophie pratique d'origine européenne. En effet, notre approche d'anthropologie philosophique à partir du corps vécu cherche à comprendre comment le corps est inhérent à la relation à l'autre et aux autres. Ensuite, cette anthropologie refuse d'opérer une séparation dualiste entre l'humain et le vivant. En effet, si nous parvenons à appréhender un corps en première personne, c'est en tant que vivant et comme vivant, nous nous saisissons nous-mêmes comme appartenant à la vie, à la communauté des vivants. Cette empathie assez évidente avec les animaux de compagnie et plus généralement les mammifères supérieurs se perd peu à peu, à mesure que nous allons vers des formes de vie moins complexes ou radicalement différentes comme celles des animaux marins, des insectes ou des unicellulaires. C'est donc à partir de cette intercompréhension corporelle que nous partageons, à des degrés plus ou moins élevés, avec les vivants que surgit la question des perspectives ou des points de vue sur le corps humain. Et d'une manière plus générale, lorsque nous réfléchissons, nous faisons fond sur ce corps vivant, sur cette conscience immanente du corps et du corps de l'autre, sur " une aptitude à ressentir en profondeur et à anticiper intensément ce qu'une conscience réflexive ne saisira que plus tard et de façon plus rationalisée » (Depraz, $2011:$ 11-12).

Notre hypothèse est pourtant que cette intercompréhension des corps vivants repose elle-même chez l'homme sur l'intercorporéité dont nous sommes tous issus : une imbrication corporelle qui précède notre naissance. La «spatialité personnelle»suppose toujours des perspectives, parfois isolées, parfois croisées. Nous parlerons des «personnes » au sens grammatical du terme, Je-tu-il, qui permettent de rendre compte à tour de rôle de la différence des perspectives, afin de souligner la relativité des points de vue et le fait que la troisième personne (il) ne devrait jamais oublier qu'elle dérive des deux premières et que la première (je), est moins originaire qu'il n'y paraît puisqu'elle suppose la deuxième (tu). Comme le français n'a plus de déclinaisons, nous devons passer un peu artificiellement par les personnes 
pour signaler les perspectives du même corps qui pourraient être marquées par l'accusatif (équivalent du corps en troisième personne ou objectivé), vocatif (corps en deuxième personne, celui de l'adresse et de la relation) et le nominatif ou corps-sujet (en première personne) ${ }^{1}$.

Sur la base de cette communauté éprouvée de vivant, entrons dans ce cercle herméneutique $\mathrm{du}$ "corps lucide», selon la belle expression de Natalie Depraz, pour aborder la question de l'espace à travers celle des perspectives du corps.

\section{LOCALISATION DU CORPS VIVANT}

Plutôt que de spatialisation au sens géométrique, nous devrions parler de localisation du corps pour souligner que, par sa présence, il ouvre l'espace homogène des corps physiques juxtaposés et ainsi transforme leur neutralité. Il bouleverse l'espace pour le rendre local et hétérogène. Ainsi, le corps vivant ouvre-t-il un lieu et un temps, c'est-à-dire qu'à partir de lui un "ici et maintenant » prennent leur sens ${ }^{2}$. Il faudrait même dire que le corps nous ouvre le temps et l'espace en moments et en lieux. Il marque l'espace et le temps de manière existentielle : ma terre natale, ma date de naissance. Notre corps est certes un corps parmi les corps, mais il est avant tout le lieu affectif ou la localisation émotionnelle, parce qu'il est un objet sensible et affectif (ce n'est qu'en lui que nous pouvons éprouver du plaisir et de la douleur, de la jouissance et de la peine) et par lequel le monde est contrasté (agréabledésagréable, désirable-indésirable, chaud-froid,...). C'est pour cette raison qu'il faudrait dire que le corps vivant n'occupe pas l'espace mais le crée en l'ouvrant. En reprenant l'opposition de Marc Augé entre l'espace anonyme comme non-lieu et le lieu anthropologique (1992), nous pourrions parler du corps comme lieu primordial. Et ce moment d'activité corporelle est le moment personnel ou existentiel de l'espace, ce qui fait que l'espace s'ouvre toujours à partir d'un lieu corporel vivant.

Les philosophies de l'existence ont particulièrement insisté sur le fait que le corps est spatial ou «en situation » en comprenant la spatialité corporelle comme la manière dont nous nous situons dans le monde et la manière dont nous nous rapportons à nous-mêmes dans cette localisation. La situation désigne donc la dimension existentielle de la localisation en insistant sur le fait que les humains sont dans une relation corporelle à eux-mêmes. Toutefois, contrairement à la position de certains existentialistes et notamment du

${ }^{1}$ Les génitif, datif et ablatif pourraient apparaître comme les cas intermédiaires entre corpssujet et corps-objet.

2 Dans le cadre de ce papier, nous n'insistons pas sur la dimension temporelle ouverte par le corps, même s'il est impossible de l'éluder complètement dès lors que nous abordons la question spatiale. 
premier Heidegger, qui privilégie le rapport du retour sur soi à celui de l'êtreau-monde (comme inauthentique ou comme dévalement), il ne s'agit pas pour nous de poser une hiérarchie entre ces deux mouvements, le mouvement transitif et le mouvement réflexif, mais plutôt de remarquer que toute réflexivité étant originairement corporelle, elle passe par une extériorité, un autre que soi qu'elle ramène à soi et phagocyte ${ }^{3}$. Ainsi, notre relation à nousmêmes, aux autres et au monde est la condition de possibilité de notre réflexivité, la condition de la découverte de soi et de la conscience de soi. C'est ainsi que nous pourrions dire $\mathrm{d}^{\prime}$ emblée que toute localisation opère en passant par l'autre. Comme une position par détour, l'autre étant le plus court chemin à soi-même.

Ce faisant, nous devons nous opposer à une longue tradition moderne, lorsque «Descartes » 4 (Marion, 2013 ; Van Cung, 2012) ouvre la modernité avec la subjectivité réfléchissante, il manque le corps et opère ainsi un double ratage pourrait-on dire: d'une part en se tenant à la première personne comprise comme seule conscience (détachée du corps), le cogito, et en traduisant ce moment subjectif fondateur en terme de substance métaphysique, comme une res cogitans, une chose pensante. Il manque donc doublement le corps en n'allant pas jusqu'au bout des conditions de son expérience de pensée : le corps vivant en première personne. En effet, s'il établit que la veille et le rêve sont indiscernables pour le rêveur lorsqu'il rêve qu'il est éveillé, en revanche, il oublie de dire que même le rêveur endormi a bien un corps. Quoi que je voie, même en rêve, j'ai bien une perspective et un lieu dans l'espace à partir duquel je rêve et "je vois", à partir duquel je pense: celle de mon corps. Mais bien avant ce tournant moderne, la philosophie grecque avait, elle aussi, imposé une inversion logique des perspectives corporelles, puisque le corps en troisième personne, qui vient toujours après mon corps propre, mon corps, par lequel toute pensée du corps est conditionnée, a été pensé avant lui et comme premier.

Pourtant, ce que le corps propre nous offre, c'est précisément une spatialité d'un autre type que celle des corps qui ne sont pas le nôtre. C'est une spatialité qui n'est pas partes extra partes, des parties extérieures les unes aux autres, $c^{\prime}$ est-à-dire qui ne répond pas au principe de la juxtaposition et de l'occupation de l'espace par les corps autour de nous, puisqu'il s'agit d'une spatialité articulée à un centre actif.

${ }^{3}$ Tandis que Heidegger rate la dimension concrète du corps, qu'il se contente de poser, Sartre accentue quant à lui la dimension d'objectivation de soi par les autres et désarticule la relation de mon corps à moi et de mon corps aux autres.

${ }^{4}$ Il faut bien entendu distinguer une réception critique, mais souvent réductrice d'un certain cartésianisme et les recherches récentes qui tentent de sortir de ces caricatures. 
Il y a une différence fondamentale entre le fait d'être dans l'espace comme l'une de ses parties, côtoyant les autres choses, et vivre spatialement, en étant conscient de vivre dans l'espace et d'être en relation à l'espace5. (Patočka, 1998 : 31)

Alors curieusement, cette condition première de vivant a dû se (re)gagner peu à peu dans l'histoire de la philosophie et sortir de l'occultation dans laquelle elle était plongée. Ainsi, refusant la conception passive du corps telle que l'ont élaborée les empiristes qui en faisaient le lieu de rassemblement des sensations, Maine de Biran (1766-1824) ouvre cette voie d'une activité et d'une subjectivité corporelles en première personne avec la notion d'effort ou de capacité à se mouvoir. Mais ce n'est donc qu'avec la phénoménologie européenne du XXème siècle (Husserl, Heidegger, Fink, Patočka ou MerleauPonty), que le corps peut apparaitre comme subjectif et actif en tant qu'il est ce à partir de quoi les objets se donnent : un objet zéro qui ouvre le monde.

Ce corps [vivant] est un objet si proche de nous qu'il n'est pas thématisé, c'est quelque chose dont nous ne faisons pas l'expérience dans notre expérience, ce que nous négligeons dans de nombreux cas. (idem : 27)

Or, ce corps est un corps spatial, qui nous rapporte au monde et aux choses parmi lesquelles il trouve sa place pour exercer son activité. Ou plutôt, il est situé au sens où la place dans laquelle il me situe et qui n'est jamais entièrement mienne, qui ne fait pas de moi une chose, au contraire des choses inertes qui sont simplement juxtaposées.

Savoir où nous sommes est une fondation nécessaire et le point de départ de la vie. (ibid.. : 32)

C'est ce paradoxe de la spatialisation non-chosale qui fait de notre corps un vivant très particulier auquel nous nous identifions, parce que nous y sommes identifiés. Le corps vivant étendu est en effet lieu et localisation parmi les choses dans le monde, d'une manière radicalement particulière, puisque l'autre dimension de la localisation est celle d'un moi, moi-même, comme subjectivité ou d'une intériorité. Dans la présence à soi, revenant toujours à lui, ce sujet corporel se rapporte à lui-même et se saisit lui-même réflexivement. Je touche mon coude gauche avec ma main droite et explore ce gonflement ressenti.

Ainsi, précisément, si mon corps est localisé globalement et en parties, c'est parce qu'il est personnel, parce qu'il n'est pas seulement un objet de perception extérieure ou l'objet d'une abstraction réflexive, mais qu'il est dans un certain sens ce que je suis. C'est en effet par lui et à travers lui que les fonctions vitales trouvent leur unité et à partir de lui que se joue mon

${ }^{5}$ Nous traduisons et nous soulignons. 
orientation dans un contexte, car me donnant une orientation, il me confère un sens.

\section{OSCILLATION ENTRE NOMINATIF ET ACCUSATIF}

En repartant de la différence acquise depuis la phénoménologie de Husserl entre le corps subjectif, le corps vivant, mon corps vécu en propre, le corps propre, et le corps objectif ou corps en troisième personne, nous voyons que le premier, le corps en tant qu'il est saisi par le sujet en première personne, est la condition de possibilité des corps objectifs. Ainsi, le corps saisi en troisième personne, est-il construit par un regard s'abstrayant du contexte et de ses conditions de possibilité. C'est le cas en sciences notamment, mais plus largement sous un regard objectivant. Pourtant ce corps second tend ensuite à s'imposer dans la longue histoire de la médecine avec les corps de l'anatomie dans la dissection post mortem (Vésale) et de la physiologie, le corps des chirurgiens épaulés par les anesthésistes, et qui s'impose aujourd'hui de plus en plus grâce à de nouvelles techniques comme l'imagerie médicale, grâce aux scanners, aux radiographies, aux échographies, etc... Et socialement, c'est l'image effectivement qui s'impose avec notre culture des écrans (photos, vidéos, télévisions, ordinateurs, smartphones et autres tablettes). C'est un corps mis à distance par l'œil et objectivé sous le regard.

Cette distinction entre le corps propre comme chair, conscience corporelle vécue et vivante (en allemand, Leib) d'une part, et le corps objectivé, corps physique dénué de conscience (en allemand, Körper) d'autre part, vient se superposer à celle de la langue anglaise qui distingue le corps vivant (body) et le corps mort (corpse). Mais si nous préférons les cas d'une langue à déclinaisons plutôt que des termes différents, c'est pour insister sur le fait que la distinction des perspectives n'implique aucune séparation, mais bien une articulation.

Ainsi, le corps vivant est celui qui métabolise et sans cesse se maintient luimême dans un rapport actif d'échange avec l'extériorité qu'il trace comme ce qui est physiquement au-delà de son individualité métabolique. Je bois, mange et respire. Pourtant, parler d'une identité métabolique, c'est aussi souligner la constante auto-altération du corps qui, n'étant jamais statique, n'est jamais identique à lui-même. Il grandit, vieillit, est marqué par la fatigue ou allégé par la lumière. Dès lors, Hans Jonas (1903-1993) affirme que le corps vivant ne peut être saisi par la biologie moléculaire, qui en se situant au niveau infra-cellulaire (celui de l'ADN et des virus) a perdu la spécificité de la vie comme souci constant et actif du maintien de soi dans l'être par un échange constant de matière ${ }^{6}$ (Jonas, 2001, 2013).

${ }^{6}$ Le terme allemand de métabolisme, Stoffwechsel, dit explicitement cet échange. 
Par son mouvement centripète, le métabolisme construit donc le corps par auto-identification. Pourtant, "aujourd'hui », c'est-à-dire depuis la double révolution industrielle et notre possibilité de projeter nos actions dans le fonctionnement de machines, nous devons encore ajouter la dimension technique du corps qui nous projette vers l'extérieur. Le corps organique s'est doté d'un corps inorganique (ma voiture qui prolonge et décuple ma capacité à me déplacer, mon œil qui se prolonge dans la caméra, etc.). Mais la révolution virtuelle déploie encore bien davantage le corps dans l'espace. Tous ces écrans et les réseaux virtuels augmentent non seulement notre corps perceptif, mais aussi communicationnel et notre mémoire. Tout se passe comme si notre modernité occidentale avait inventé, avant de l'exporter à l'échelle mondiale, un corps inorganique planétaire qui englobe et surplombe les corps individuels vivants en les connectant. Dès lors, il n'est plus possible de parler du monde naturel face à nous, mais de cet espace créé par l'homme, un espace naturel-artificiel, un écoumène (Berque, 2009). Nous y sommes, pendant un moment, un point de capiton du réseau globalisé, qui ne connaît ni centre, ni périphérie : un réseau complexe, qui connaît plusieurs niveaux et une altération constante.

\section{COÏNCIDENCE À SOI}

Si mon corps vivant est compris comme perspective de mes perspectives successives, c'est-à-dire la perspective de l'ici et du maintenant source de mon expérience à partir de laquelle j'appréhende forcément les autres vivants, les autres humains et le monde, perspective à partir de laquelle nous pouvons changer de perspectives, c'est parce qu'il est pourtant insubstituable. Il est notre point ce zéro d'expérience, celui à partir duquel s'ouvre toujours et chaque jour le monde comme monde orienté. Où que je me déplace dans le monde ou dans l'espace, mon corps me situe à partir d'une asymétrie spatiale radicale, qui fait que je vois ce qui est devant moi et pas ce qui se trouve dans mon dos; qui fait que ma droite se trouve toujours dans la même latéralité même si je tourne sur moi-même. De même, le haut et le bas sont toujours relatifs à cette ouverture corporelle, puisque je peux me retrouver la tête en bas ou même couché, mais ils sont toujours liés à un positionnement corporel, relatif cette fois à la gravité que subit ma chair qui est portée par le monde de la vie. C'est que mon corps est-au-monde, orienté dans un monde terrestre qui est d'ailleurs polarisé et centré7.

7 La condition des hommes dans l'espace est gagnée par arrachement à la condition de terrien, car la terre reste la « base » des astronautes. 
On pourrait évidemment remarquer que le terme français lui-même $\mathrm{d}^{\prime}$ orientation ${ }^{8}$ se réfère déjà à une présence du corps au monde, au soleil levant qui point toujours du même côté. Mon corps est donc la condition de possibilité de toute perspective subjective, mais dans une relation avec le monde qui le conditionne à son tour. Mon corps spatialise et rend ainsi l'espace radicalement hétérogène. Dès lors, c'est par abstraction et dans un second temps que nous pensons l'espace homogène, géométrique et purement étendu.

Pour le dire encore autrement, mon corps constitue un pli (dimension réflexive) dans l'espace à partir duquel il se déploie (perspective des perspectives). C'est pour cette raison que Jan Patočka (1907-1977) parle du corps qui se donne comme horizon plutôt que comme présence. C'est-à-dire $q^{\prime}$ 'il se donne comme ne se donnant pas objectivement ou en face-à-face. Il est le point noir à partir duquel la lumière peut jaillir, point noir qui se laisse donc oublier et gommer, mais comme l'est le fond de l'œil à la vue, condition du regard porté sur toute chose et sur tout objet.

Par sa présence constante au monde, mon corps semble se faire oublier pour laisser la place à ce qu'il perçoit, à l'objet perçu, éloigné sous le regard. Un objet en troisième personne. Ainsi, sa présence qui se donnerait avant tout comme une activité, un dynamisme peut-elle s'objectiver peu à peu, même si elle n'apparaît jamais comme un objet saisissable de tous côtés, sous tous les points de vue. Par contre, c'est précisément grâce à ce mouvement dynamique $\mathrm{du}$ corps (sujet de perspectives) que des objets peuvent m'apparaître en totalité (selon toutes les perspectives).

Le phénomène du Je comme objectif est toujours seulement partiel, nous ne pouvons jamais nous envelopper nous-mêmes dans un champ phénoménal comme nous enveloppons les objets, comme nous enveloppons l'autre Je (Patočka, op.cit. : 51).

Or, cet horizon n'est pas statique. Mon corps est la base de mon dynamisme, du mouvement de mon être, de mon apparaître et de ma manifestation, de mes propres activités et des possibilités qu'il projette et des effets qu'il produit. Il est donc un objet kinesthésique aussi, puisque, comme y a insisté Merleau-Ponty, en lui l'activité et la passivité coïncident et que je peux toucher mon propre corps dans un mouvement réflexif d'activité et de passivité.

Notre corps est une vie qui est spatiale en elle-même, produisant elle-même sa localisation et se spatialisant elle-même. (idem : 31)

8 De nombreuses langues européennes partagent cette référence à l'Orient pour désigner la position du corps terrien par rapport au soleil levant. Or, peu à peu, cette position relative va se perdre et le terme va acquérir un statut de positionnement absolu. Alors, l'Occident pourra désigner une région du monde au même titre que l'Orient. 
Alors, nous pouvons relire la spatialité du corps humain comme liée à notre condition de terrien. Directement liés au dynamisme de mon corps, le haut et le bas renvoient pour ainsi dire à un effort ou du moins une résistance à la gravité terrestre. La droite et la gauche renvoient à la symétrie de nos mouvements corporels, de nos membres et de nos organes des sens qui s'offrent par deux dans cette symétrie pour ouvrir le monde en profondeur. L'avant et l'arrière quant à eux donnent la direction de notre activité dans le monde, comme une prise sur lui et une activité à l'œuvre.

Le dynamisme primordial, tel que nous en faisons l'expérience, caractérise la spatialité de notre présence physique. (ibid. : 41)

C'est dans notre être-au-monde que le corps se donne, lorsqu'il est aux autres, aux choses et au monde bien plus que lorsqu'il se réfléchit. Il faut donc souligner l'articulation et le renvoi mutuel de la kinesthésie (le mouvement de notre corps) et de l'esthétique (la perception des objets).

\section{MOUVEMENT VERS SOI}

Revenons sur le fait que cette perspective n'est pas interchangeable. Mon corps est mien, par le rapport dialectique (métabolique) qu'il instaure avec son milieu et procède ainsi à sa propre individualisation en traçant une frontière organique au-delà de laquelle commence le non-moi. Dès lors, c' est à partir du corps organique que s'ouvrent l'extériorité et l'altérité. Comme ouverture précisément. Et comme ce rapport avec la limite est toujours aussi celui de son franchissement, le corps se donne simultanément comme ouverture et fermeture à son milieu, comme investi et intègre, comme tout à la fois prothétique et inviolable ( $d^{\prime}$ où les questions $\mathrm{d}^{\prime}$ implantation, de remplacement de ses organes, ...).

C'est aussi à partir de mon corps et de son processus métabolique que je peux éprouver la notion d'intériorité, de subjectivité et ainsi aussi d'extériorité, de milieu. L'intériorité comme pensée ou conscience, comme for intérieur, ne serait que le dérivé tard-venu de l'extériorité du corps.

Ce corps mien est aussi actif dans une présence qui se donne sous la forme d'une capacité ou d'un "je peux » (Merleau-Ponty), d'un désir-persévérance (le conatus de Spinoza) ou d'un élan vers le monde (Bergson), grâce auquel nous disposons de capacités (innées comme la vision ou acquises comme la capacité de jouer du violon). Cette disposition est immédiate et, dans les meilleurs des cas, identique au mouvement de mon être, mais lorsque mon corps ne répond pas immédiatement, adéquatement ou totalement à cet élan du moi, lorsqu'il lui résiste et fait obstacle (par la maladie, la vieillesse ou le handicap), il s'en dissocie et devient un autre. Alors, dans un vécu continu, il commence à devenir un objet pour moi. Il est peut-être significatif que Maine 
de Biran qui a tant insisté sur l'identité corporelle dans sa jeunesse, ait peu à peu décrit l'expérience corporelle comme duelle, comme ce corps qui semble se distancier de moi à force de me résister et de me contrarier en vieillissant.

Pourtant, il faudrait ajouter que si notre corps semble se dissocier peu à peu de nous-même avec la vieillesse et les résistances invalidantes qu'elle impose parfois progressivement à notre volonté, le mouvement de l'existence semble aller vers une coïncidence à soi, par-delà le corps qui nous entrave. Comme si ce don initial devait peu à peu se résorber dans un mouvement de rapprochement à soi-même par lequel on se l'approprie. Si l'on nait avec un corps reçu, le mouvement de notre histoire nous permet de nous lui appartenir ou de nous en dissocier. Les corps qui rayonnent n'ont pas une beauté plastique, mais sans doute l'éclat de la coïncidence avec son histoire. Dans les meilleurs des cas bien sûr, la coïncidence peut être totale. Par contre, certaines histoires mènent à une déchirure de soi, dans le sens où les signes objectifs nous arrachent à nous-mêmes. Les corps alors semblent ternes et gris, marqués par cette entrave. C'est ainsi qu'un corps qui était la condition de possibilité de mon existence devient son support. Avec toutes les questions relative à la mort juste que cela implique.

\section{ARTICULATIONS ET CROISEMENTS DES PERSPECTIVES}

Pourtant, notre perspective est sans doute plus liée encore aux autres vivants et aux autres humains qu'à la terre, car elle est toujours relative et $s^{\prime}$ inscrit toujours parmi d'autres qui la relativisent. Mon point de vue n'est pas celui du regard que je croise, ce qui fait que les orientations s'articulent entre elles par le déplacement de cette perspective des perspectives dans l'espace qu'elle a ouvert.

Ce croisement de perspective s'éprouve d'ailleurs à l'égard de son propre corps. En effet, ce corps est mien, car je peux entretenir avec lui un rapport de relative altérité ou de plus ou moins intense identification. Dans sa formule classique, Gabriel Marcel décrit cette relation ambiguë et souligne que «J'ai un corps et je suis mon corps ». C'est ainsi que, dans une articulation des perspectives, je peux regarder mon corps vivant, au moins partiellement. Je regarde mes jambes ou mes bras, mes mains. Bien sûr, pour regarder mon propre visage, je devrai, tel Narcisse, passer par le biais du reflet et par celui de la photographie, même si dans le "selfy ", je la prends moi-même du bout de mon bras avec mon téléphone portable. Et pour voir mon dos ou des parties de mon corps que je n'ai jamais vues de cette manière, je devrai passer par des ruses afin de gagner quelque distance. Songeons au Christ mort de Mantegna, corps humain dans une posture impossible pour l'autoportrait. Ce corps est mort certes, au double sens où la vie l'a quitté, mais aussi où il figure la perspective radicale de la troisième personne. Il présente une distance radicale et une perspective inaccessible en première personne. 
$C^{\prime}$ est sans doute une relation de réciprocité ou de miroitement réciproque plus qu'une relation comprise analogiquement. Les autres voient en nous des objets comme nous voyons en eux un dynamisme interne. En présence mais pas comme donné. "Je vois et je suis vu. J'intègre ce miroitement en moi » (Patočka, op.cit. : 52), mais ce regard est précédé toujours d'une réciprocité corporelle. "Cette mutualité est le fondement des structures sans lesquelles le langage serait incompréhensible» (idem : 52).

Certes, l'autre m'objective d'une certaine manière et se voir exclusivement à travers les yeux des autres, c'est se perdre comme je. Sur ce point, Sartre à raison, mais l'autre n'est pas seulement un « $\mathrm{Il}$ », il est aussi, originairement, altérité relationnelle, un « tu ». S'arracher au regard objectivant de l'autre, c'est le premier moment de la réflexivité. C'est alors que la relation devient consciente sur le mode de la présence à soi explicite et plus seulement sur le mode implicite ou de l'oubli de soi comme dans l'effort dynamique au sein du monde (Patočka, ibid.. : 51).

Sans que ces deux perspectives opposées sur mon corps vivant se désarticulent, mon corps est ainsi caractérisé par cette double face d'activité et de passivité, de subjectivité et d'objectivité, de corps pour moi-même ou pour les autres, qui permet de le qualifier en première et en troisième personne. Et ces deux faces peuvent être simultanées ou alternatives.

Aucune de ces perspectives n'étant entièrement clôturée sur elle-même ou exclusive des autres, elles s'articulent entre elles de manière dynamique dans la pratique. C'est d'ailleurs de cette complexité que surgissent de nombreuses questions éthiques contemporaines importantes. Est-ce que les parties détachées (cheveux, reins, cellules souches, gamètes, embryons,...) ou détachables (sang, sperme,...) de mon corps m'appartiennent, au sens où je les possède même lorsque je n'en dispose plus ? Est-ce que je les possède parce que j'en dispose ? Parce qu'elles proviennent d'un corps global dont elles sont détachées? Certes cette distance à l'égard des parties de mon corps est d'autant plus problématique qu'elle peut être investie par des agents qui la transforment et en modifient le sens, notamment en lui donnant une valeur économique 9 . Peut-on mélanger les corps pour les guérir ou les améliorer? Peut-on injecter en eux des parties et des substances issues d'autres corps, humains ou animaux, organiques ou mécaniques, vivants ou morts? Le corps qui semble tracer lui-même sa frontière est-il ainsi violé ou amplifié par davantage d'altérité que celle qu'il métabolise spontanément?

$C^{\prime}$ est aussi dans mon rapport aux autres que je peux vouloir ou consentir à me livrer comme objet corporel. Dans la protection que je lui offre en raison de ma force physique ou ma seule présence rassurante. Mais aussi par exemple de manière temporaire lors de la grossesse, lorsque le corps se laisse traverser

${ }_{9}^{9}$ Certains juristes français (comme Marie-Angèle Hermitte) ont d'ailleurs proposé de penser le rapport aux parties détachées du corps à partir du droit de propriété intellectuelle pour souligner leur caractère inaliénable. 
et habiter par un autre corps ou lorsque dans l'amour physique par exemple, j'accepte et désire être le corps de la jouissance de l'autre, l'objet de ses phantasmes. Alors se pose la question de prêter son corps pour le projet de maternité d'une autre femme, moyennant rémunération ou pas. Mais le corps peut être aussi concédé en totalité (grossesse pour autrui) ou en partie (avec le don de sang ou de sperme) sans préjudice pour ma vie. Mais je peux aussi céder des parties non renouvelables comme avec le don d'organes (reins) ou même post-mortem le don de mon corps pour la recherche ou pour la transplantation. En dehors du contexte médical, je peux céder mon image pour des raisons esthétiques ou commerciales (publicité) ou pour soutenir des causes (mais dans ce cas, mon image n'est efficace que comme symbole de mon action). Ce point d'équilibre au sein du paradoxe corporel est fragile.

En effet, lorsque je cesse $d^{\prime}$ adopter une position d'objet par mon libre consentement, je deviens une « chose » pour l'autre ou un « matériau » en vue d'un usage (parfois technique) au détriment de ma subjectivité (vol d'organes, esclavage, exploitation de la force de travail ou sexuelle, traite d'êtres humains, vol d'enfants,...). Alors, la dynamique de la relation intersubjective, qui se décline dans la réciprocité, l'alternance et l'oscillation des volontés ou des désirs aura fait place à la violence du rapport de forces ou de possession, unilatéral et statique. Alors que les relations humaines incarnées supposent toujours que l'autre puisse être aussi - mais jamais exclusivement - un objet corporel dans un rapport de réciprocité, d'oscillation et d'alternance, la violence, au contraire, consiste à dépouiller l'autre de son initiative de sujet, donc de son activité et du mouvement de son être.

Pour résumer. Si mon corps avec lequel je coïncide est pourtant distant par rapport à moi, c'est dans un quadruple sens. D'une part, se maintenant luimême sans cesse dans l'être, il ne coïncide jamais identiquement avec luimême. Ensuite, il est tout à la fois sujet et objet de mes sens (toucher, vue, ouïe,...) ; par ailleurs, je peux me l'approprier davantage par des pratiques et ainsi je peux réduire sa résistance et l'adapter à mon désir (par des entraînements sportifs, des répétitions chorégraphiques, apprentissages pour l'utilisation de machines...); enfin, et en sens inverse, je peux mettre, partiellement ou temporairement, mon corps à disposition des autres (ma voix, ma force physique, mon intelligence,...). Dans ces quatre types de distance, le corps subjectif et le corps objectif sont articulés comme alternatifs et même parfois situés dans un continuum, voire simultanés.

\section{ATTACHEMENT INTERCORPOREL}

Au-delà de la dimension perceptive, venons-en maintenant à la dimension affective et interpersonnelle du corps vivant. Partant de la communauté des vivants, nous pouvons attribuer l'intercorporéité humaine dans le monde commun au fait que tous les corps naissent des corps, puisque les corps se 
produisent entre eux. Dépendant physiquement, psychiquement et affectivement des proches qui les accueillent, les nouveaux-venus apparaissent d'emblée comme des êtres relationnels, qui sont accueillis et pris en charge en raison de leur existence vulnérable ${ }^{10}$ (Barbaras, 2011).

Dans cette mesure, Jan Patočka écrit que le premier mouvement de l'existence tient à un enracinement corrélatif de l'accueil qui nous est réservé par les autres et qui nous permet d'accéder au monde, d'être-au-monde parmi les autres avant d'être dans le monde de la nature ou des objets.

Nous voici arrivés à la perspective de la deuxième personne, celle de l'adresse et du vocatif, à celle qui conditionne les deux autres. Ce corps de l'enracinement n'est-il pas, en effet, en raison de la proximité charnelle, celui du tu ? Toujours adressé et dépouillé ? Celui de l'altérité vulnérable que vise Levinas $^{11}$ ? Ce corps sensible de l'autre en deuxième personne n'est en effet ni totalement subjectif ou dynamique (comme un «je»), ni totalement objectif et donné (comme un «il») : il est le corps de la présence immédiate aux besoins concrets de l'autre.

Le vivant comprend le vivant, le vivant humain et le vivant animal, en particulier les mammifères. Ainsi, ce ne sont pas seulement les corps vivants en général qui bénéficient d'une intercompréhension, parce qu'ils partagent l'existence (Burgat, 2012). Et cette intercompréhension repose sur une expérience originaire, une empathie liée au fait qu'ils se sont portés entre eux à l'existence par la gestation. Alors avant la relation duelle, il faudrait souligner la fusion dont ils doivent se départir. Seule la séparation préalable permettra une relation véritable.

Ce corps relationnel se prolonge au-delà que ce sont les autres qui nous accueillent à notre naissance. Comme l'a fait remarquer le psychanalyste Donald Winnicott, parce que le lien qui nous rattache à l'autre est premier, l'espace est d'emblée transitionnel pour le nouveau-né. L'espace est donc initialement relationnel. Certes, si le plus souvent une "mère suffisamment bonne » porte son enfant dans le monde, l'accueil est malheureusement parfois vécu sur le mode paradoxal de l'abandon, il peut être froid ou inadéquat, et même traumatisant ou violent. Pourtant, comme il a toujours déjà eu lieu, il opère un mouvement de localisation du nouveau-né parmi les autres. C'est dans la mesure où les autres, nos proches, nos parents ou leurs substituts, prennent (plus ou moins bien) en charge nos besoins physiologiques, nos besoins de corps vivant et métabolisant, que nous pouvons nous attacher à eux et à un lieu relationnel, et dès lors développer un corps et une existence affective qui deviennent peu à peu autonomes, mais

${ }^{10} \mathrm{Il}$ faudrait approfondir cette vulnérabilité de la vie pour ne pas la limiter à la question des besoins corporels.

11 Si Levinas insiste sur le face-à-face et la parole d'autrui, nous insistons ici davantage sur la dimension générique de cette altérité et donc sur le mouvement de la genèse des corps séparés. 
toujours interdépendants et vulnérables ${ }^{12}($ Arendt, 1983). Le monde s'ouvre de proche en proche à partir des autres dans lesquels notre existence s'enracine. Comme l'écrit Patočka : « Nous sommes en relation à nous-mêmes en étant en relation à l'autre et à des choses de plus en plus nombreuses et finalement à l'univers comme tel, nous situant ainsi dans le monde » (op.cit. : 31).

Mais c'est sous la plume d'une phénoménologue, que, en faisant un pas en arrière, nous trouvons les sources de cet attachement-enracinement avec l'expérience intérieure du corps préalable à la naissance de celui qui devient un autre, avec un attachement intra-utérin : la grossesse. La grossesse du point de vue de la mère dévoile une dimension plus intime encore de ce corps vivant.

Comment mon corps est-il capable de donner naissance à un individu radicalement singulier et irréductiblement différent de tout autre (y compris de ceux dont il procède), tout en croissant depuis l'intimité la plus intime de ma chair, depuis la profondeur la plus abyssale de mon désir intérieur ? En produisant cette dépossession de moi-même qui est exposition à l'autre, l'enfant me reconduit à l'espace intérieur de ma chair émotionnelle. (Depraz, 2011 : 31)

Si bien sûr, cette expérience est davantage donnée aux femmes qui ont connu une ou plusieurs grossesses dans leur chair, elle apparaît indirectement à tous ceux et celles qui l'observent en tant que spectateurs proches ou semiacteurs.

Etant un objet sensible et affectif, mon corps est ainsi ce qui m'attache aux autres qui m'accueillent, mais ce corps m'a été donné par les autres corps et " la confiance à l'égard du monde comprend toujours l'être avec les autres » (Patočka, op.cit. : 50). Ce premier mouvement de l'existence, le mouvement d'enracinement, d'ancrage suppose la mise à couvert des existences dans l'abri sécurisant d'autres vies bienveillantes et bienfaisantes. Celles-ci, en effet, prennent en charge les besoins liés à la vie nue du nouveau-né, à son existence corporelle et créent des relations singulières et uniques avec lui. Ce mouvement d'enracinement qui a lieu initialement dans la petite enfance avec l'amour parental, ou du moins l'amour des adultes de la génération précédente, se répète à l'âge adulte avec l'amour érotique et les liens d'une famille dont nous avons l'initiative. En outre, tout au long de la vie, ce sont les corps des proches qui nous mettent à couvert, à l'abri du besoin et de la misère, par leur présence régulière et répétée. Au point que l'on peut y voir « un seul corps »: la famille et les amis proches, ce sont toutes ces parties de moi qui vivent hors de moi.

Pourtant, là encore, si cette expérience est universelle comme celle de la grossesse, c'est dans la mesure où elle présente une expérience singulière et

$12 \mathrm{H}$. Arendt parle aussi de ces besoins fondamentaux du corps qui nous inscrivent dans une maisonnée, mais elle en souligne uniquement la dimension laborieuse. 
insubstituable ${ }^{13}$. Marqué par le fait que la vie lui a été donnée de la coexistence avec un autre corps, le corps humain se donne en outre sous une perspective particulière, puisqu'il est sexué et donc toujours déjà marqué par une relativité et une partialité. Marqué par l'attirance sexuelle et « orienté » sexuellement, il éprouve la relation à l'autre en son cœur.

$C^{\prime}$ est en deuxième personne que les corps se nourrissent entre eux ou s'affament et se dessèchent entre eux. Bien sûr, il faut insister sur le fait que cette ouverture initiale de fait peut ensuite être accentuée ou amoindrie par le type de rapport aux autres que nous entretenons. Et en ce sens, l'espace sera un facteur d'éloignement ou de rapprochement des corps qui prennent concrètement soin les uns des autres selon les mouvements d'existence (naissance, croissance, rencontres, séparation, attirances sexuelles, fréquentations, collaborations, relations de proximité, ...). C'est donc à partir de ce dynamisme corporel qu'il faut aussi comprendre les relations intersubjectives comme relations aux autres corps : lorsque certains corps-tu s'éloignent au point de se perdre comme «tu » et de devenir des « ils » dans le monde des objets en troisième personne.

C'est ainsi dans ce mouvement d'enracinement primordial que les corps se donnent en deuxième personne, étant dans une zone intermédiaire entre la subjectivité de la première personne et l'objectivité de la troisième.

[...] le positionnement de soi-même dans le monde a la structure d'un Je-Tu. Nietzsche dit que le tu est plus ancien que le Je. [...] Le Je personnel est toujours le corrélatif d'un Tu. (Patočka, idem:36)

Ne pourrait-on pas dire que la prise en considération de la dimension radicalement relationnelle du corps est le signe de la deuxième personne ? Relativement subjectif ou relativement objectif, toujours adressé à l'autre parce qu'il fut l'objet d'une sollicitude, le corps apparaît avant tout comme relationnel. Un corps purement en troisième personne, un corps en dehors de toute relation, serait le résultat d'un arrachement de tous ses liens préalables et de toutes ses conditions de possibilité, abstrait intellectuellement ou pratiquement et considéré pour lui-même, délié. En y regardant de plus près alors, c'est cette intercorporéité qui est la source oubliée de la corporéité propre et neutre, du je et du il, donc, par transitivité, la condition première de la corporéité en troisième personne et de notre relation au monde. La condition oubliée de l'espace du corps.

Dans un tout autre cadre, celui de la théorie critique, Axel Honneth a quant à lui montré, en se basant d'ailleurs sur les travaux de Winnicott et de Mead, que cette dimension relationnelle du corps vivant est à la base de la confiance intersubjective que nous développons à l'égard de nous-mêmes et des autres.

${ }^{13}$ Nous opposons le singulier à l'universel et le particulier au global comme deux types de polarités différentes. 
$C^{\prime}$ est en effet à ce premier niveau de reconnaissance, celui de la confiance, celui de la reconnaissance affective au sein de la sphère de l'amour et de l'amitié qui prennent en charge les besoins les plus élémentaires du corps et prémunissent contre toute violence ou maltraitance physique, que peut se développer un premier rapport affectif positif à soi-même et aux autres, un rapport moral décisif pour l'individuation et la socialisation (Honneth, 2000).

A l'autre extrémité de la vie, pour l'expérience de la mort de l'autre, lorsque son corps se referme définitivement, c'est une partie de moi-même qui s'en va. Lorsque l'être cher meurt, me voilà amputé de cette partie de moi qu'il était. Et avec son départ, c'est une partie de mon monde qui s'éteint, c'est ma chair qui se rétrécit d'autant (Ricœur, 2007). L'enchevêtrement n'est plus ici physiologique, mais affectif et émotionnel.

\section{BIBLIOGRAPHIE}

Hannah ARENDT, La condition de l'homme moderne, Paris, Calmann-Levy, 1983.

Marc AUGÉ, Non-lieux, introduction à une anthropologie de la surmodernité, Paris, Seuil, 1992.

Renaud BARBARAS, La vie lacunaire, Paris, Vrin, 2011.

Augustin BERQUE, Ecoumène. Introduction à l'étude des milieux humains, Paris, Belin, 2009.

Florence BURGAT, Une autre existence. La condition animale, Paris, Albin Michel, 2012.

Natalie DEPRAZ, "L'autre intime. Phénoménologie de la grossesse », in Sylvain CAMILLERI et Christophe PERRIN, Epreuves de la vie et souffrance d'existence, Argenteuil, Le Cercle Herméneutique, 2011.

Axel HONNETH, Lutte pour la reconnaissance, Paris, Cerf, 2000.

Hans JONAS, Le phénomène de la vie, Bruxelles, De Boeck, 2001.

Hans JONAS, Essais philosophiques, Paris, Vrin, 2013.

Jean-Luc MARION, Sur la pensée passive de Descartes, Paris, PUF, 2013

Jan PATOCKA, Body, Community, Language, World, trans. E. Kohak, Chicago and La Salle, Open Court, 1998.

Paul RICOEUR, Vivant jusqu'à la mort, Paris, Seuil, 2007.

Kim Sang Ong VAN CUNG, L'Objet de nos pensées. Descartes et l'intentionnalité, Paris, Vrin, 2012. 
RÉSUMÉ : Cet article montre que le corps ouvre l'espace, parce qu'il est localisé dans un monde et que cette localisation repose sur le croisement et les juxtapositions de trois perspectives: le corps propre, le corps objet et le corps relationnel. Notre hypothèse est que les corps sont d'abord enchevêtrés et superposés en tant qu'ils sont vivants et relationnels, à la deuxième personne. Et la dimension subjective, d'un moi isolé, ne se gagne que progressivement, tandis que la dimension totalement objective résulte d'une abstraction.

MOTS-CLÉS : perspectives, intercorporéité des vivants, intercorporéité humaine, phénoménologie, corps propre, grossesse. 